\title{
Thyroid-Stimulating Hormone Receptor Autoimmunity and Local Factors in Multiple Risk Factors Are Mainly Involved in the Occurrence of Pretibial Myxedema
}

\author{
Changgui Lan ${ }^{\mathrm{a}, \mathrm{e}}$, Liping Hu${ }^{\mathrm{a}}$, Chengqi Liao ${ }^{\mathrm{a}}$, Yuhong Shi ${ }^{\mathrm{b}}$, Yi Wang ${ }^{\mathrm{a}}$, \\ Shuanghua Cheng ${ }^{c}$, Wei Huang ${ }^{d}$
}

\begin{abstract}
Background: Pretibial myxedema (PTM) is a local mucinous dermopathy associated with thyroid diseases. Since the etiology of PTM is unclear, the aim of this study is to identify the risk factors for PTM and their etiological roles in PTM occurrence.

Methods: A large-scale case-control study $(\mathrm{n}=1,200)$ was performed to identify risk factors for PTM by calculating odds ratio (OR) values and $95 \%$ confidential intervals. The PTM group entered a glucocorticoid treatment trial. Patients with complete response were followed up to the first relapse in a cohort study. The relative risk (RR) values of the main risk factors were calculated for PTM relapse to test their etiological roles.

Results: Among the 19 factors, six risk factors were identified: thyroid-stimulating hormone (TSH) receptor antibody (TRAb) (OR 42.93), autoimmune thyroid disease (AITD) or AITD history (OR 10.30), local trauma (OR 6.55), venous stasis posture (OR 6.16), cigarette smoking (OR 4.48), and age (OR 1.05). Serum TRAb levels were positively correlated with the severity of PTM. Of note, 371/400 patients received glucocorticoid treatment, and 330 achieved complete response. The serum TRAb levels after treatment decreased dramatically compared with those before treatment. After stopping
\end{abstract}

Manuscript submitted September 16, 2020, accepted October 7, 2020

Published online November 3, 2020

aDepartment of Dermatology, The Second Affiliated Hospital of Chengdu Medical College, China National Nuclear Corporation (CNNC) 416 Hospital, Chengdu, China

${ }^{b}$ Department of Nuclear Medicine, The Second Affiliated Hospital of Chengdu Medical College, China National Nuclear Corporation (CNNC) 416 Hospital, Chengdu, China

'Department of Pathology, The Second Affiliated Hospital of Chengdu Medical College, China National Nuclear Corporation (CNNC) 416 Hospital, Chengdu, China

${ }^{\mathrm{d}}$ Department of Health Checkup Center, The Second Affiliated Hospital of Chengdu Medical College, China National Nuclear Corporation (CNNC) 416 Hospital, Chengdu, China

${ }^{e}$ Corresponding Author: Changgui Lan, Department of Dermatology, The Second Affiliated Hospital of Chengdu Medical College, China National Nuclear Corporation (CNNC) 416 Hospital, No.4 Er Huan Lu Bei Si Duan, Chengdu City, China. Email: lcg638212@163.com

doi: https://doi.org/10.14740/jocmr4352 glucocorticoid treatment, serum TRAb levels increased significantly when PTM relapsed $(\mathrm{P}<0.001)$. In 165 relapse cases, an increase in serum TRAb levels occurred first, followed by persistent venous stasis posture or local trauma and finally PTM. The RR of elevated serum TRAb levels was 6.73 in PTM relapse cases. In the elevated serum TRAb level group, the RRs of local trauma, venous stasis posture, and local trauma plus venous stasis posture were 8.81, 6.5, and 8.84, respectively, for PTM relapse cases.

Conclusions: TSHR autoimmunity and local factors in the six identified risk factors are the main causes of PTM occurrence.

Keywords: Pretibial myxedema; Etiology; TSH receptor autoantibody; Local skin trauma; Venous stasis posture; Case-control study; Glucocorticoid treatment; Cohort study

\section{Introduction}

Pretibial myxedema (PTM) is a localized myxedema often occurring in the pretibial area, which differentiates it from generalized myxedema (a synonym of hypothyroidism). Since it was first reported in 1895 [1], PTM has been considered as a complication of Graves' disease (GD, which was first described in 1835) and represents one component of the extra-thyroidal triad (exophthalmos, pretibial myxedema, and acropachy) [2]. PTM occurs in about $1.7 \%$ of GD cases [3], and it has also been found to occur occasionally in patients with Hashimoto's thyroiditis (HT) and idiopathic hypothyroidism (IH) [4, 5]. Therefore, it is also referred to as thyroid dermopathy or thyroid-associated dermopathy.

Clinically, most PTM cases occur after patients are diagnosed with autoimmune thyroid diseases (AITDs), often at the skin of the lower legs and feet, and seldom at other skin sites outside the lower extremities. Lesions are circumscribed, non-pitting, and solid or hardened, ranging from nodules, diffuse swelling, and plaques to large tumors and elephant skinlike legs and hands. In the active stage, the lesions start out as erythematous and gradually enlarge. Sometimes when lesions grow to a certain extent, they enter a stable stage for a time period during which they no longer enlarge. A few gradually become hardened and enter a sclerotic stage, and in even fewer 
cases, nodule variants disappear spontaneously [6].

Histopathologically, PTM is characterized by significant thickening of the dermis due to excessive deposits of mucopolysaccharides (such as hyaluronan (HA) and chondroitin sulfate) [7]. Hence, PTM is a localized mucinosis resulting from extracellular matrix remodeling as a result of excessive HA deposition. However, its etiology and pathogenesis are currently unclear. To our knowledge, four etiological hypotheses have been proposed. First, dysfunction of the thyroid gland is a possible cause of PTM because many PTM cases occur in patients with hyperthyroidism or hypothyroidism. Rapid reversion from the hyperthyroid state to the euthyroid state has been hypothesized to be a factor precipitating PTM. However, this hypothesis was rejected as more and more PTM cases have been found to occur in euthyroid patients. Second, overproduction of thyroidstimulating hormone (TSH) in the pituitary gland has been proposed to be a common factor in the causation of exophthalmos and PTM. However, this hypothesis is not supported by the fact that PTM often occurs in patients with hyperthyroidism whose serum TSH levels are lower than those of normal individuals. Third, autoimmune pathogenesis has been proposed because of two observations. One is that the thyroid diseases (GD, HT, and $\mathrm{IH}$ ) with PTM as a potential complication are a group of AITDs in which TSH receptors (TSHRs) become autoantigens, and autoantibodies to the autoantigens occur [8]. The other is that this group of AITDs has elevated serum TSH receptor antibodies (TRAbs), while PTM always has elevated serum TRAb [9, 10]. The serum immunoglobulin $\mathrm{G}$ (IgG) of PTM patients has been reported to stimulate cultured primary fibroblasts of leg dermis to produce HA [11]. In vitro experiments have found that the TSH stimulating antibody (TSAb) subtype of TRAbs may mimic the action of TSH to stimulate orbital fibroblasts to produce HA through the PI3K-AKT signaling pathway [12]. It has been thought that TRAb is the causative agent of thyroid gland and extrathyroidal manifestations in GD. However, simple autoimmune theory cannot explain a lower prevalence of PTM in AITD. PTM's preference for the lower legs, ascribed to site-specific properties of fibroblasts, has not been confirmed because PTM can occur at anatomical sites outside of the lower legs. Finally, multi-factorial etiology has been postulated in which local factors (e.g., local trauma and prolonged standing) are superimposed on systemic connective tissue inflammation caused by autoimmunity to TSHR, which might transform a subclinical inflammation into a clinical entity [13].

As PTM is a rare dermopathy, the above-mentioned hypotheses have never been tested by any large-sample epidemiological studies. Here, we first designed a large-scale casecontrol study to identify the risk factors of PTM. Then, the identified main risk factors were tested with an immunosuppressive glucocorticoid treatment intervention and a cohort study in PTM patients with complete response.

\section{Materials and Methods}

\section{Participants}

Participants were as follows: PTM patients who visited the De- partment of Dermatology; GD without PTM (GD w/o PTM) patients who visited the Departments of Nuclear Medicine, Endocrinology and Pediatrics; and normal persons who visited the Health Checkup Center and Department of Pediatrics in the Chinese National Nuclear Corporation (CNNC) 416 Hospital from July 1, 2013 to September 30, 2018.

A definite diagnosis for PTM was made under the background of thyroid diseases or thyroid disease history, the presence of typical skin lesions, or atypical skin lesions (atypical locations and atypical appearances) plus histopathology of mucinous degeneration and positive Alcian blue staining in the dermis (modified from [6] and [14]). GD was diagnosed on the basis of clinical and/or biochemical evidence of thyrotoxicosis plus one or more of the following features: serum TRAb positive, thyroid-associated ophthalmopathy and/or dermopathy and/or acropachy, hypoechoic and increase in vascularity shown by thyroid ultrasound with Doppler, and diffuse elevated radioactive iodine uptake (RAIU). Biochemical evidence of thyrotoxicosis is an elevated serum triiodothyronine level (T3 $>2.79 \mathrm{nmol} / \mathrm{L}$, normal value $0.92-2.79 \mathrm{nmol} / \mathrm{L}$ ) and undetectable serum TSH level $(<0.01 \mathrm{mU} / \mathrm{L})[15,16]$. The definition of normal persons is that they have no symptoms or signs and have no abnormal findings in physical examinations and laboratory tests for thyroid, heart, liver, lung, kidney, and brain function.

\section{Ethical issues and informed consent}

All of the participants provided written informed consent to participate in this study. The study was approved by the Ethics Review Board of the CNNC 416 Hospital; and this study was conducted in compliance with the Declaration of Helsinki.

\section{Study design}

A case-control study $(1: 2)$ was designed to identify the risk factors for PTM among 19 investigated factors. The control group included GD w/o PTM patients and normal persons in a ratio of $1: 1$, and the sex ratios $(\mathrm{M} / \mathrm{F})$ were respectively $1: 3$ and $1: 1$. We performed a correlation analysis between the severity of PTM and serum TRAb levels and administered immunosuppressive glucocorticoid intralesional injection treatment to test the causal relationship between TRAb and PTM in this PTM group. Finally, local factor exposures and PTM relapses were analyzed in a cohort study to test if local factors and TRAb were the cause of PTM relapse (Fig. 1).

\section{Selection of cases and controls}

The participants were eligible if they met the following inclusion criteria and did not meet the exclusion criteria.

\section{Inclusion criteria}

Participants were included in the corresponding groups if they 


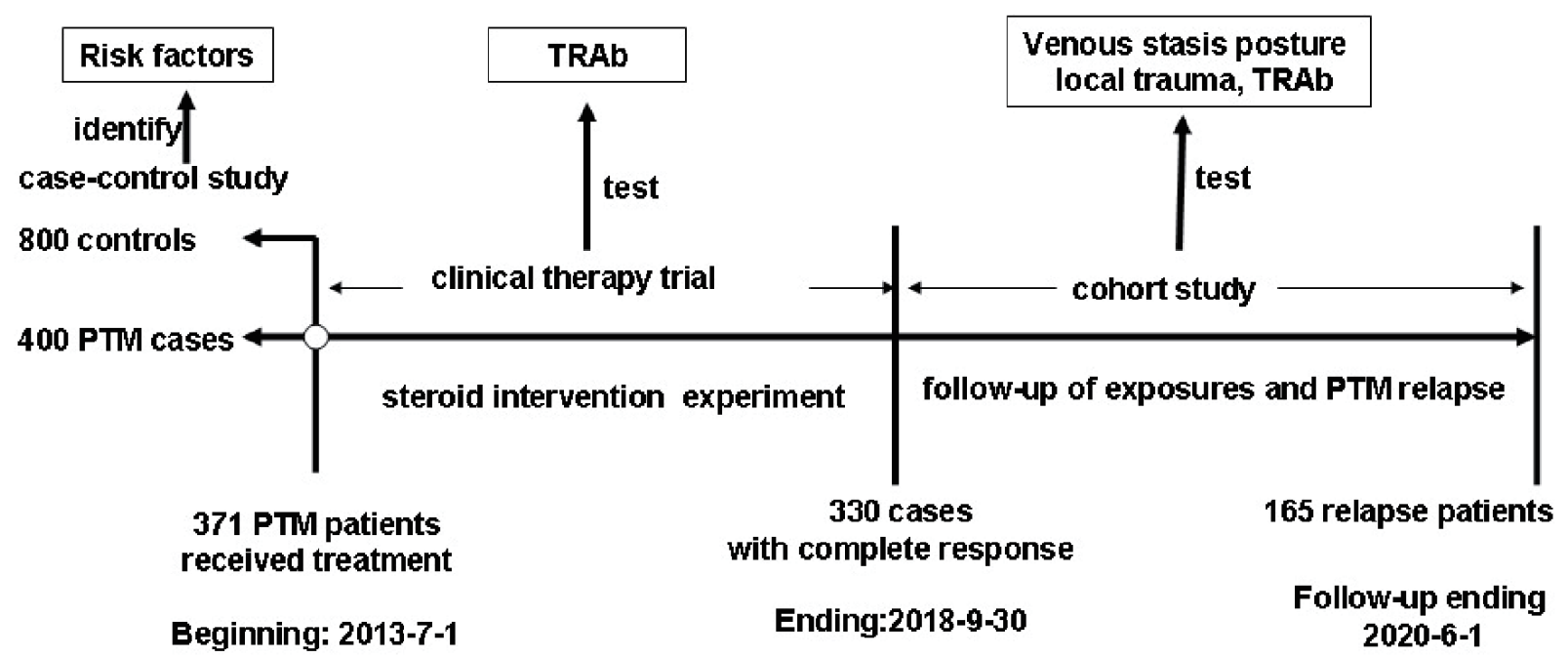

Figure 1. Study design for identifying and validating the risk factors for PTM occurrence. PTM: pretibial myxedema; TRAb: TSH receptor antibodies; TSH: thyroid-stimulating hormone.

met one of the following sets of inclusion criteria: 1) meeting diagnostic criteria of PTM, GD w/o PTM, and normal persons; 2) first visiting the CNNC 416 Hospital for diagnosis or therapy.

\section{Exclusion criteria}

Participants were excluded if they met one of the following criteria: 1) no written informed consent to participate in the study; 2) did not provide his/her medical history, physical examination, blood, or skin tissue if needed; or 3) had no integral data of medical history, physical examination, and lab tests if needed.

The final numbers of participants of GD w/o PTM and normal persons chosen from eligible participants were respectively equal to the PTM group (Fig. 2).

\section{Factors and definitions}

The 19 investigated factors included age (at the onset of PTM and GD), sex, occupation (1 year before the onset of PTM and GD), cigarette smoking (continuous or cumulative smoking for $\geq 6$ months before PTM and GD), alcohol drinking (at least $40 \mathrm{~g}$ of liquor in the 30 days before PTM and GD), local trauma (closed or open skin trauma at lesional sites within 3 months before PTM and GD), venous stasis posture (persistent standing/sitting/squatting posture with immobility $\geq 4 \mathrm{~h} /$ day before PTM and GD), AITD/AITD history (thyroid symptoms, signs, and positive thyroid autoantibodies, or thyroid autoantibodies alone, mainly including GD, HT, and IH), exophthalmos (exophthalmometry scale $>20 \mathrm{~mm}$ ), acropachy (digital clubbing of digits and toes), I-131 treatment, thyroid function (serum T3, T4, free T3 (FT3), free T4 (FT4), and TSH), and serum thyroid autoantibodies (TRAb IU/L, thyroid peroxidase antibody (TPOAb) IU/mL, and thyroglobulin antibody (TgAb)
$\mathrm{IU} / \mathrm{ml})$.

\section{Measurements of serum thyroid hormones and thyroid autoantibodies}

The serum levels of thyroid hormones (total T3 (TT3) nmol/L, FT3 pmol/L, total T4 (TT4) nmol/L, and FT4 pmol/L) and $\mathrm{TSH} \mathrm{mIU} / \mathrm{L}$ ) were measured with direct chemiluminescence (ADVIA Centaur XP immunoassay system, Siemens Healthineers, Germany). The serum levels of TgAbs, TPOAbs, and TSHR-binding inhibitory immunoglobulins (TBIIs, also called TRAbs) were measured with an electrochemiluminescence immunoassay (anti-TSHR, Tg-Ab, and TPO-Ab kits using Elecsys and Cobas e 601 analyzers, Roche Diagnostics $\mathrm{GmbH}$, Germany) according to the manufacturer's instructions.

\section{Statistical analysis}

SPSS 17.0 statistical software package was used (SPSS Inc., Chicago, IL, USA). Continuous variables were described as means \pm standard deviation (SD). Categorical variables were described as percentages (\%). Comparisons of means were performed by independent or paired sample $t$-tests. The paired Wilcoxon rank sum test was used to compare non-parametric variables. Comparison of percentages was done by the Chisquared test. When binary logistic regression analysis was performed, categorical variables except occupation were divided into yes (assign " 1 ") and no (assign " 0 "). TRAb levels in continuous variables were divided into three sections, which included < 1.75 IU/L (assign “1"), 1.75 - 39.99 IU/L (assign “2”), and $\geq 40 \mathrm{IU} / \mathrm{L}$ (assign “ 3 ”). Binary logistic regression analysis was used to calculate the odds ratio (OR) and 95\% confidence interval $(\mathrm{CI})$ of the relationship between independent variables and PTM. Relative risk (RR) values and their 95\% CI were calculated through crosstabs. Statistical significance was set at 


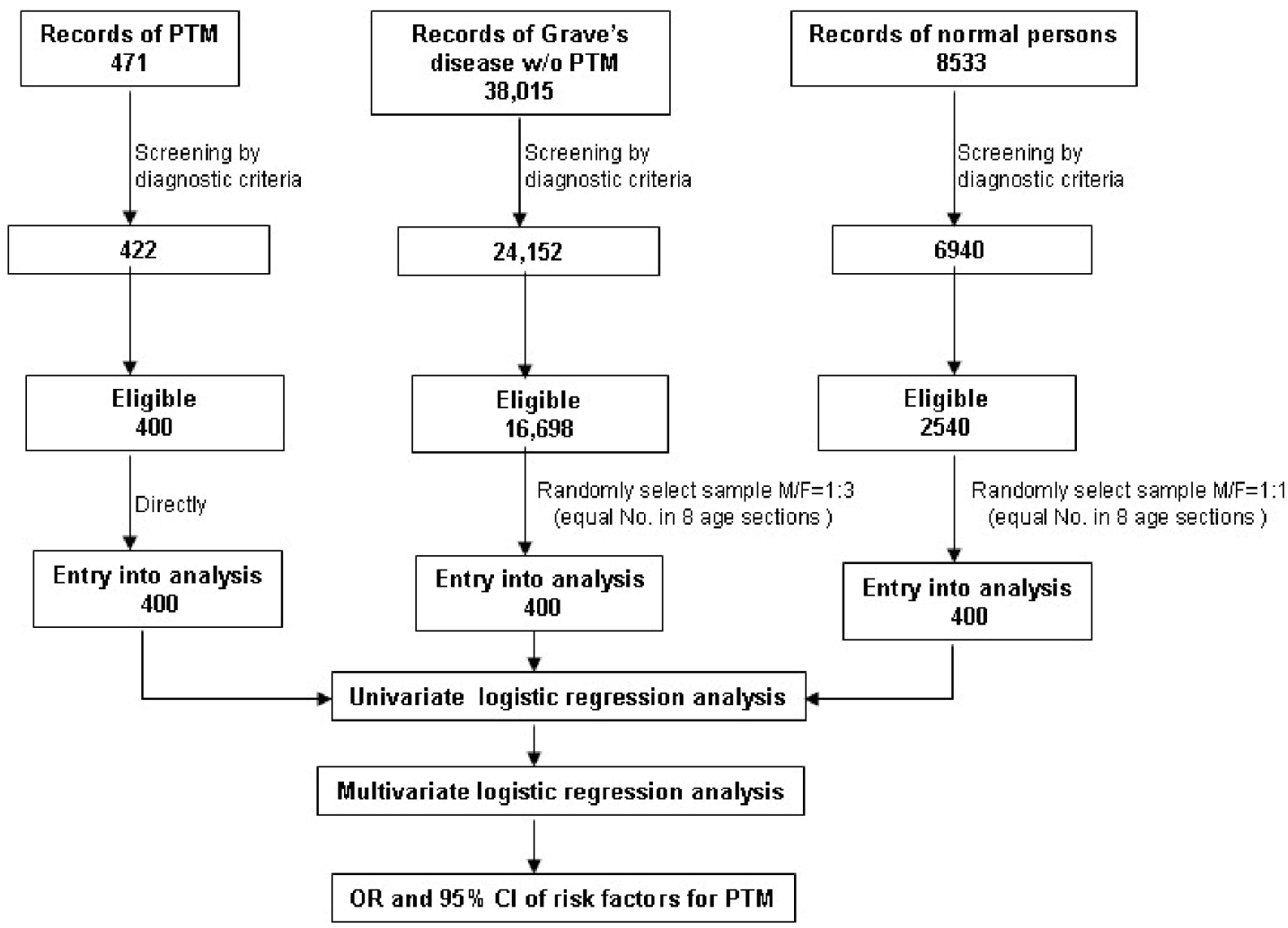

Figure 2. Flowchart of participant selection and risk factor analysis in the case-control study. OR: odds ratio. Cl: confidence interval. PTM: pretibial myxedema.

$\mathrm{P}<0.05$, and significant difference was set at $\mathrm{P}<0.01$.

\section{Results}

General characteristics and the distribution of 19 investigated factors among PTM cases, GD w/o PTM cases, and normal persons

Based on the diagnostic criteria, we obtained 422 PTM cases, 24,152 GD w/o PTM cases, and 6,940 normal cases from a total of 471 records of PTM patients, 38,015 records of GD w/o PTM patients, and 8,533 records of normal persons in the CNNC 416 Hospital from July 1, 2013, to September 30, 2018. Both inclusion and exclusion criteria were used to screen these cases again, and 400 PTM cases, 16,698 GD w/o PTM cases, and 2,540 normal cases were eligible for the study. All 400 eligible PTM cases served as the case group and entered final statistical analysis. A 1:2 ratio of case-control (1 case: $1 \mathrm{GD}$ w/o PTM: 1 normal person) was taken into consideration. Therefore, each of the 400 cases was, respectively and randomly, selected from both eligible GD w/o PTM group and eligible normal person group according to a 1:3 (GD w/o PTM) and 1:1 (normal person) male/female ratio to represent their corresponding populations. Finally, 1,200 cases were subjected to statistical analysis.

The age of PTM occurrence was $45.7 \pm 12.2(16-86)$ years old, and the sex ratio was 1:0.91 (M/F). PTM occurred among multiple occupations and unemployed persons. Cigarette smoking and alcohol drinking were $36.8 \%$ and $34 \%$, respectively. Before PTM occurrence, $23.5 \%$ cases had local skin trauma, and $71.5 \%$ had persistent venous stasis posture; $98.8 \%$ of cases had AITD history or AITD. All cases had elevated serum TRAb levels. Associated exophthalmos and acropachy occurred in $71.5 \%$ and $14.3 \%$, respectively. Interestingly, $67.3 \%$ cases had received I-131 treatment. Serum levels of thyroid hormones, TSH, and antithyroid autoantibodies in PTM cases are shown in Table 1. In comparisons of the factors between the PTM and control groups, there were no consistent results.

\section{Thyroid autoimmunity and local factors were the main risk factors for PTM occurrence}

The relationships of the 19 investigated factors with PTM analyzed with univariate and multivariate logistic regression are shown in Table 2. Serum levels of thyroid hormones (serum FT3, FT4, TT3, and TT4), TSH, TPOAb, and TgAb as well as exophthalmos, acropachy, I-131 treatment, occupation, and alcohol were unrelated to the occurrence of PTM. TRAb was the leading risk factor, and OR and 95\% CI were 42.928 (23.757, 


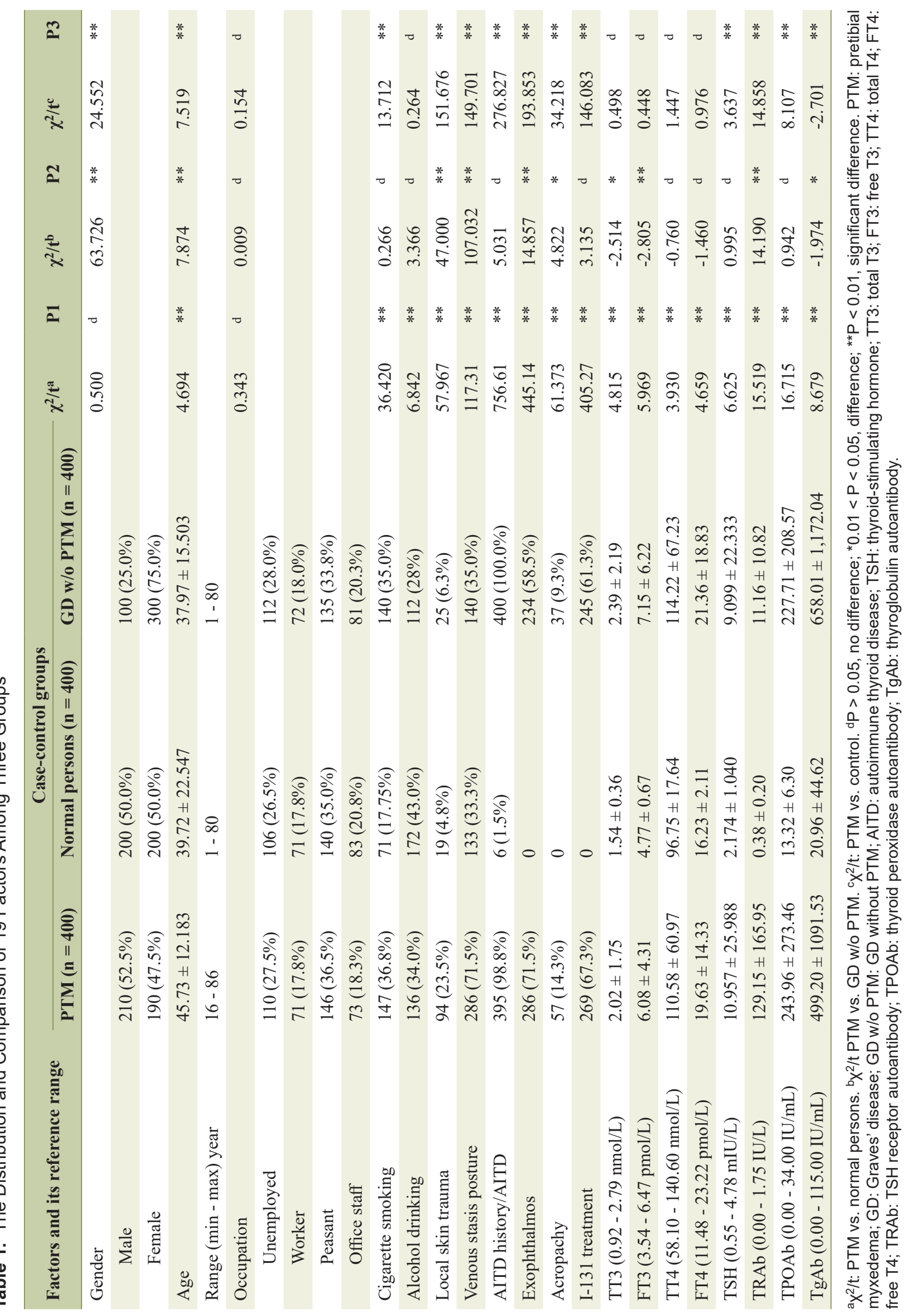


Table 2. Univariate and Multivariate Logistic Regression Analysis of 19 Factors Among PTM Patients, GD W/O PTM Patients and Normal Persons $(\mathrm{N}=1,200)$

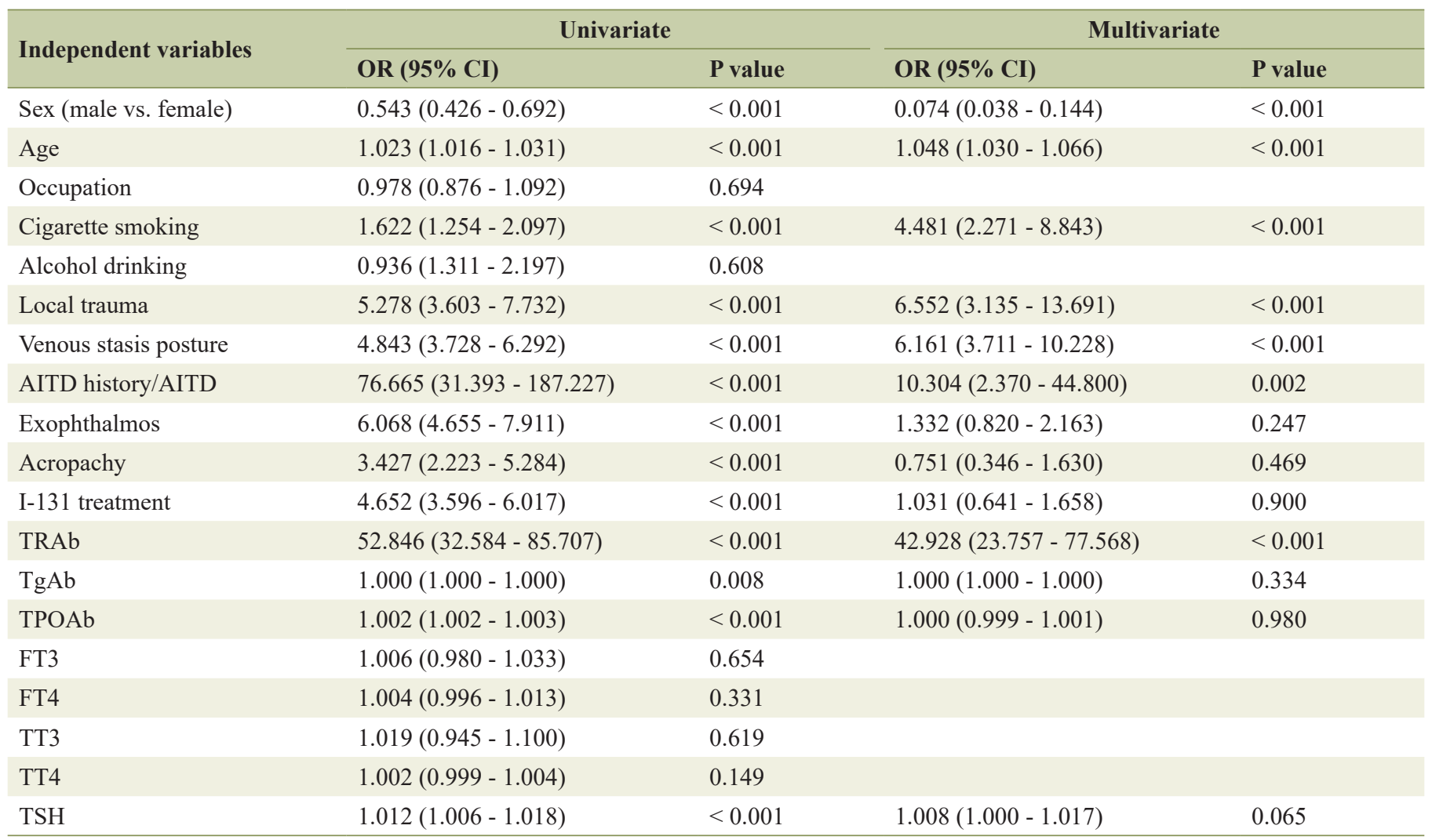

Factors adjusted for in the multivariate analysis: sex, occupation, cigarette smoking, alcohol drinking, local trauma, venous stasis posture, AITD history/AITD, exophthalmos, acropachy, I-131 treatment and TRAb. OR: odds ratio; Cl: confidence interval; PTM: pretibial myxedema; GD: Graves' disease; GD w/o PTM: GD without PTM; AITD: autoimmune thyroid disease; TSH: thyroid-stimulating hormone; TT3: total T3; FT3: free T3; TT4: total T4; FT4: free T4; TRAb: TSH receptor autoantibody; TPOAb: thyroid peroxidase autoantibody; TgAb: thyroglobulin autoantibody.

77.568). In the PTM group, 98.8\% (395/400) of cases had thyroid disease history or thyroid diseases. Of note, $328(82 \%)$ of cases had thyroid history before PTM occurrence, including 324 cases with GD history, three cases with $\mathrm{IH}$, and one case with HT. The intervals of time from AITD to PTM were 33.24 \pm 46.01 months. Sixty-seven cases were concurrent with GD. Thus, 395 cases were associated with AITD. The remaining five $(1.2 \%)$ cases occurred before thyroid disease. In laboratory examinations before PTM occurrence, the serum TRAb levels of these five patients were found to be 40.80, 80.00, 67.00, 126.00, and 168.00 IU/L (normal value 0 - $1.75 \mathrm{IU} / \mathrm{L}$ ), respectively. However, in the normal person group, seven cases had thyroid disease history, six of which were AITDs. In the GD w/o PTM group, all 400 cases had GD history. The second risk factor was AITD or AITD history $(10.30(2.370,44.800))$. The third and fourth were local trauma $6.552(3.135,13.691)$ and venous stasis posture $(6.161(3.711,10.228))$, which are both local factors. OR and 95\% CI of cigarette smoking and age were $4.481(2.271,8.843)$ and $1.048(1.030,1.066)$, respectively. Although sex ratio was statistically significant among the groups, there was no significance between PTM cases and normal persons $\left(\chi^{2}=0.5, \mathrm{n}=800, \mathrm{P}=0.479\right)$, Therefore, TRAb, AITD, AITD history, local trauma, venous stasis pos- ture, cigarette smoking, and age were potential risk factors for PTM development.

\section{Serum TRAb levels were positively correlated with the se- verity of PTM}

Since TRAb was a leading risk factor for PTM occurrence, we set out first to explore the etiological role of TRAb in PTM. Because serum TRAb levels in 400 PTM patients were significantly higher than those in $400 \mathrm{GD}$ w/o PTM patients and 400 normal persons ( $P<0.001$, Fig. 3 ), the serum TRAb level was obviously related to PTM. According to morphology and size of lesions, 400 PTM patients were divided into six groups, namely, nodules (79), diffuse non-pitting swelling (148), plaques (112), tumors ( $>2 \mathrm{~cm}$ in diameter and $>1 \mathrm{~cm}$ at height) (19), mixed (16), and elephantiasis (26) groups (Fig. $4 a)$. Variants also had differences in lesional volumes. The elephantiasis group had the largest and most serious lesions and the highest TRAb levels. The patients in the nodules group were the least ill and had the lowest TRAb levels. Serum TRAb levels were positively correlated with PTM severity (Fig. 4b), demonstrating an approximate dose-response relationship be- 


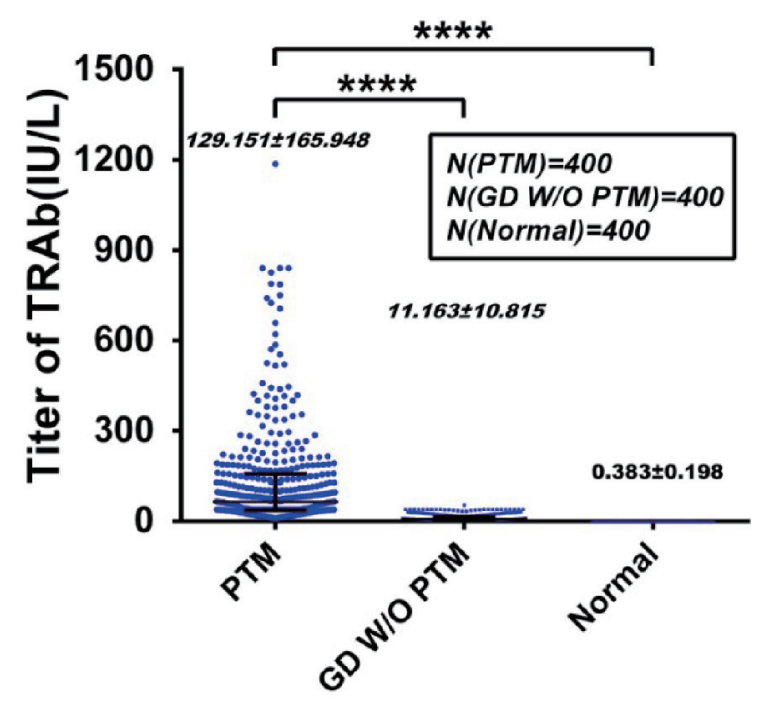

Figure 3. Serum TRAb levels in PTM patients higher than Graves' disease without PTM (GD w/o PTM) patients and normal persons. Comparison of serum TRAb levels was performed between 400 PTM cases and 800 control cases. The control cases included 400 GD w/o PTM cases and 400 normal person cases. Serum TRAb levels (129.151 $\pm 165.948 \mathrm{IU} / \mathrm{L})$ in PTM group were significantly higher than those $(11.163 \pm 10.815 \mathrm{IU} / \mathrm{L})$ in GD w/o PTM patients and those $(0.383 \pm$ $0.198 \mathrm{IU} / \mathrm{L})$ in normal persons $(P<0.001)$. The serum TRAb levels $(11.163 \pm 10.815 \mathrm{IU} / \mathrm{L})$ in GD w/o PTM cases were significantly higher than those $(0.383 \pm 0.198 \mathrm{IU} / \mathrm{L})$ in normal persons $(P<0.01)$. PTM pretibial myxedema; TRAb: TSH receptor antibodies; TSH: thyroidstimulating hormone.

tween serum TRAb levels and lesion volumes.

\section{Lowering serum TRAb levels improved PTM by gluco- corticoid treatment, and a serum TRAb level increase resulted in PTM relapse after stopping glucocorticoids}

After PTM diagnosis, 371/400 PTM patients received treatment with multipoint intralesional injection of $40 \mathrm{mg}$ triamcinolone acetonide acetate with $2 \%$ lidocaine hydrochloride (5 $\mathrm{mL}$ ), once a week. Twenty-nine (7.25\%) patients did not receive the treatment. The treatment persisted until PTM lesions were no longer changed. Of note, $330(88.95 \%)$ cases achieved complete response (lesions disappeared completely) (containing 11 tumor PTM patients treated with surgery + subdermal injection of glucocorticoid at surgical sites), and 41 (11.05\%) had major response $(\geq 80 \%$ of lesions disappeared but not completely) (Fig. 5). Serum TRAb levels (56.45 $\pm 79.21 \mathrm{IU} /$ $\mathrm{mL}$ ) after the first treatment session decreased dramatically compared with the serum TRAb levels $(130.80 \pm 165.61 \mathrm{IU} /$ $\mathrm{mL}$ ) before treatment. The serum TRAb values after glucocorticoid treatment decreased by $84.40 \pm 123.48 \mathrm{IU} / \mathrm{mL}(\mathrm{n}=$ 330 , paired $t=9.422, \mathrm{P}<0.001$ ). However, in one patient with complete response, her serum TRAb level went to $409 \mathrm{IU} / \mathrm{L}$ from 252.9 IU/L after treatment due to severe Grave's ophthalmopathy (GO) and hyperthyroidism relapses. In the follow-up (2- 7 years) of 330 cases with complete response, 165 (50\%) cases had relapses. The relapse cases received the same as the first glucocorticoid treatment session. In total, 165 cases had 477 relapses by June 1,2020. The time intervals from the end of the last treatment session to PTM recurrence were $300 \pm 293$ days (14 days to 77 months). The serum TRAb levels (58.93 $\pm 93.96 \mathrm{IU} / \mathrm{L}$ ) after the final treatment session decreased significantly compared with the serum TRAb levels (187.49 \pm 203.78 IU/L) before the first treatment. Serum TRAb levels decreased by $128.57 \pm 172.48 \mathrm{IU} / \mathrm{L}$ after repeated glucocorticoid treatment sessions $(\mathrm{n}=62, t=5.869, \mathrm{P}<0.001)$ (Fig. 6). Some TRAb levels decreased to normal levels and the relapse stopped. However, serum TRAb levels in two relapse cases remained re-ascending to $39.42 \mathrm{IU} / \mathrm{L}$ and $43.00 \mathrm{IU} / \mathrm{L}$, although their TRAb levels had decreased to normal values $(0.92$ and $1.4 \mathrm{IU} / \mathrm{L})$ after treatment. After the first treatment session, serum TRAb levels $(59.85 \pm 81.12 \mathrm{IU} / \mathrm{L})$ in the recurrence group were higher than those $(30.44 \pm 62.10 \mathrm{IU} / \mathrm{L})$ in the non-recurrence group $(\mathrm{n}=330, t=2.14, \mathrm{P}=0.033)$. Serum $\mathrm{TRAb}$ levels increased by $51.18 \pm 115.99 \mathrm{IU} / \mathrm{L}$ from those $(59.23 \pm$ $70.06 \mathrm{IU} / \mathrm{L}$ ) after last therapy to $110.41 \pm 123.78 \mathrm{IU} / \mathrm{L}$ before the next therapy when PTM first relapsed $(\mathrm{n}=165, t=-3.796$, $\mathrm{P}<0.001$ ) (Fig. 7). This demonstrated immunosuppressive glucocorticoids can treat PTM through the reduction of serum TRAb levels. When the TRAb levels increased after stopping glucocorticoids, PTM relapsed.

\section{Local factors precipitated PTM relapse under the back- ground of TRAb}

The relationship between local factor exposure and PTM relapse for different serum TRAb levels in 330 patients with complete response were followed up from the end of the first treatment session to the first relapse. The results clearly showed that TRAb persisted as positive in the blood of all 330 cases. In the 165 relapsed cases, increased serum TRAb levels occurred first followed by a persistent venous stasis posture or local trauma, and finally PTM. The period from local trauma to PTM ranged from 10 days to 2 months.

In the follow-up of 330 cases with complete response, serum TRAb levels in 13 cases had decreased to normal values $(0.3-1.75 \mathrm{IU} / \mathrm{L})$, and in 317 cases they were elevated $(>1.75$ IU/L). The relapse rate (51.74\%) of PTM in the elevated serum TRAb level group was significantly higher than that (7.69\%) in the normal TRAb level group $(\mathrm{RR}=6.73, \mathrm{P}=0.003)$. Of note, 233 cases had exposure to venous stasis posture and local skin injury, but 97 had no exposure. The relationship between local factor exposures and relapse is shown in Table 3. In the elevated serum TRAb level group, 221 cases were exposed to local factors, and 155 cases $(70.14 \%)$ relapsed. Ninety-six cases were unexposed to local factors, but nine cases relapsed and 87 cases had no relapse. The RR values of trauma, venous stasis posture, and trauma plus venous stasis posture were $8.81,6.5$, and 8.84 , respectively. These data further confirmed that local risk factors precipitated PTM relapse under the background of TRAb. In the normal serum TRAb level group, only one case recurred when 12 cases were exposed to local factors, and one case was unexposed to local factors and had no relapse. However, the only one relapse case had also an elevated 

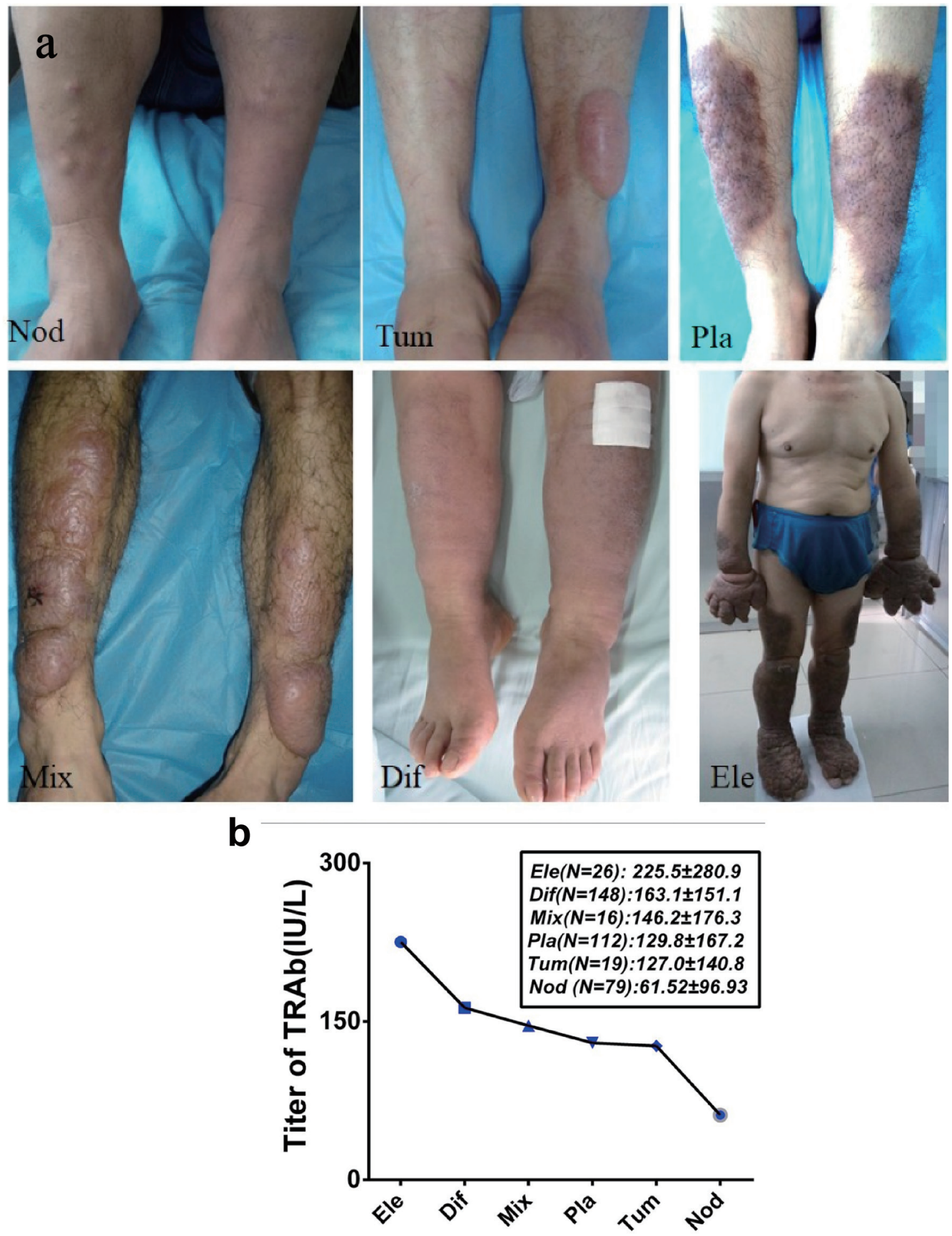

Figure 4. Positive correlation between serum TRAb levels and PTM severity. (a) Six variants of PTM lesions consisted of nodular (Nod), tumorous (Tum), plaque-like (Pla), mixture (Mix), diffuse (Dif), and elephantiasic (Ele) variants. The volume of nodule variant was the smallest, and the elephantiasic variant was the largest. (b) Serum TRAb levels in six PTM variants were positively correlated with the severity of PTM lesions. Serum TRAb levels were the lowest $(61.52 \pm 96.93 \mathrm{IU} / \mathrm{L})$ in the nodular variant and the highest $(225.5 \pm 280.9 \mathrm{IU} / \mathrm{L})$ in the elephantiasic variant $(P<0.001)$. The serum TRAb levels gradually increased with the volume increase of PTM lesions $(r=0.81, P<0.01)$. PTM: pretibial myxedema; TRAb: TSH receptor antibodies; TSH: thyroidstimulating hormone. 
before therapy

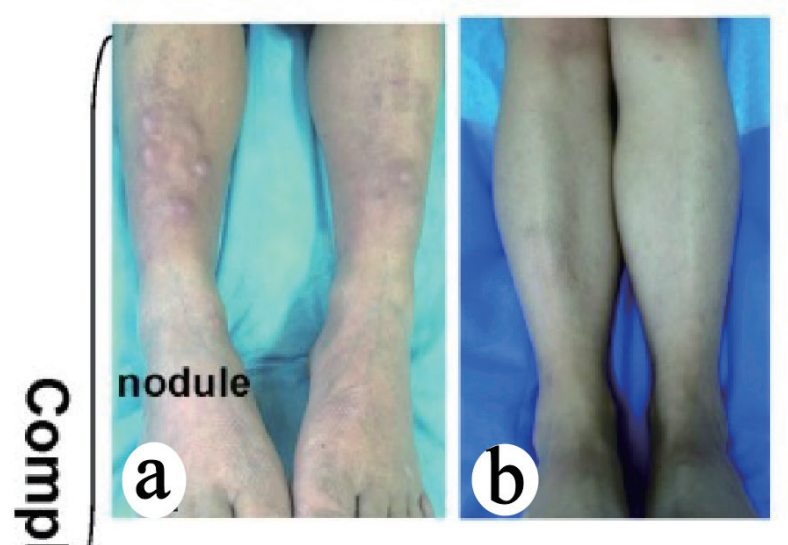

before therapy

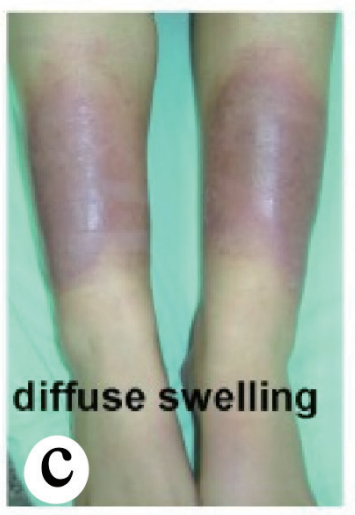

after therapy
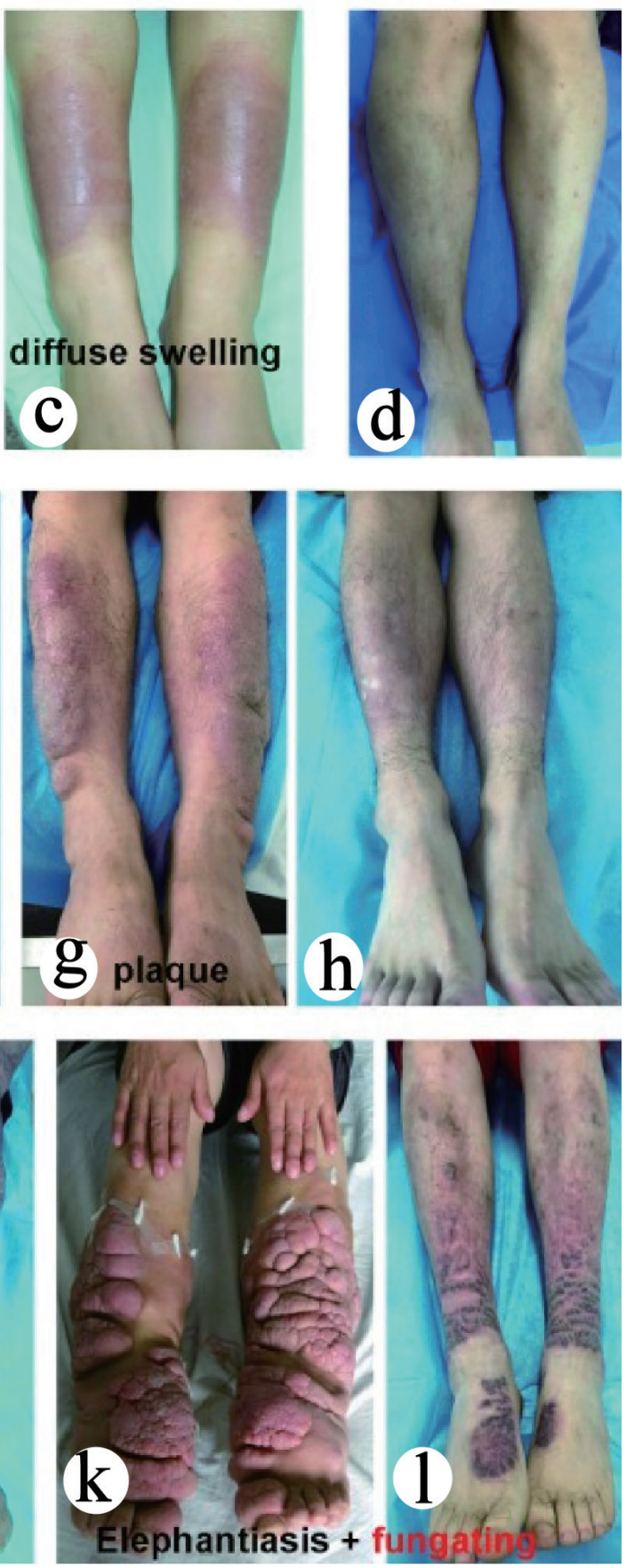

Figure 5. Significant improvement of PTM with multipoint intralesional injection glucocorticoid treatment. In 371 glucocorticoid therapy cases, the injection frequency in the nodule, plaque, diffuse swelling and mixed variants was once a week. The treatment of elephantiasic variant was divided into three stages in 1.5 to 2 years. The first stage was once a week for two to three treatment sessions, once a month in the second stage for 6 months, and once every 3 months in the third stage for 6 months. The tumor variant was treated with surgery + subdermal injection of glucocorticoid at surgical sites. Finally, 330 cases achieved complete response and 41 cases achieved major response. The cases with complete response included nodule (61 cases), plaque (99 cases), diffuse swelling (124 cases), mixed (16 cases), tumor (11 cases) and elephantiasis (19 cases). (a, c, e and g) PTM variants before glucocorticoid therapy. (b, d, f, and h) Complete response of PTM variants after glucocorticoid therapy. Forty-one cases with major response included plaque (13 cases), diffuse swelling (21 cases) and elephantiasis (seven cases). (i, k) Elephantiasis with polypoid and elephantiasis with fungating appearance before glucocorticoid therapy, respectively. (j, I) The major response after glucocorticoid therapy. PTM: pretibial myxedema. 


\section{Serum TRAb levels decrease associated wi th regression of PTM}

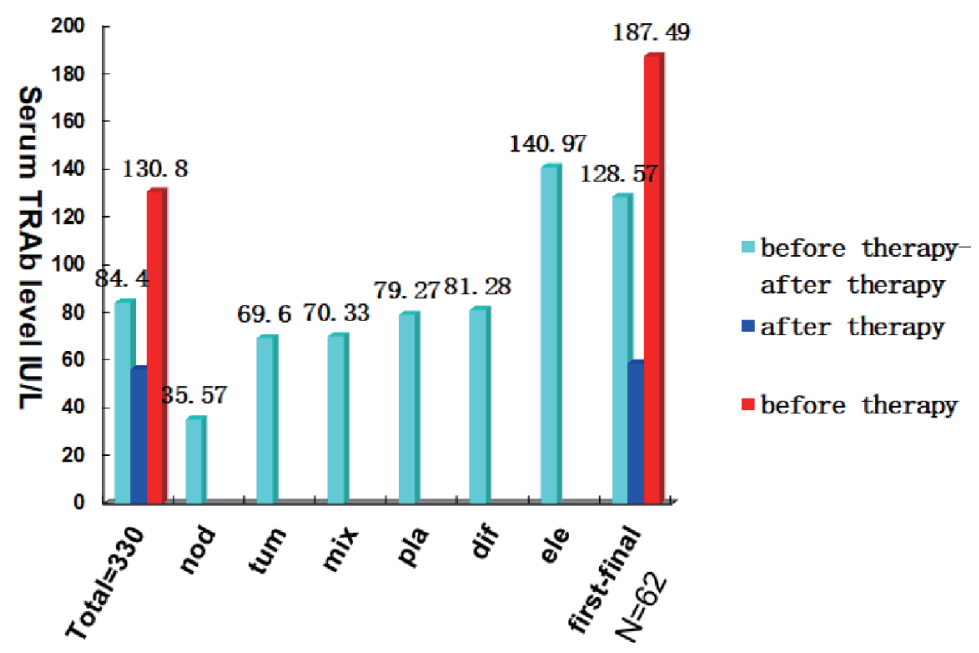

Figure 6. PTM remission related to decrease of serum TRAb levels with glucocorticoid treatment. In 330 cases with complete response, serum TRAb levels $(56.45 \pm 79.21 \mathrm{IU} / \mathrm{mL})$ after therapy decreased by $84.40 \pm 123.48 \mathrm{IU} / \mathrm{L}$ compared with those $(130.80$ $\pm 165.61 \mathrm{IU} / \mathrm{mL}$ ) before therapy (paired $t=9.422, \mathrm{P}<0.001$ ). Serum TRAb values of six PTM variants after therapy decreased by $35.57 \pm 86.80 \mathrm{IU} / \mathrm{L}$ (nodule, 61), $79.27 \pm 123.54 \mathrm{IU} / \mathrm{L}$ (plaque, 99), $81.28 \pm 130.07 \mathrm{IU} / \mathrm{L}$ (diffuse swelling, 124), $70.33 \pm 61.05 \mathrm{IU} / \mathrm{L}$ (mixed, 16), $69.60 \pm 68.68 \mathrm{IU} / \mathrm{L}$ (tumor, 11), and 140.97 $\pm 130.72 \mathrm{IU} / \mathrm{L}$ (elephantiasis, 19), respectively (paired sample $t$ test, $\mathrm{P}<$ 0.001 ). In 62 cases with repeated (two to four) relapses, serum TRAb levels decreased by $128.57 \pm 172.48 \mathrm{IU} / \mathrm{L}$ from $187.49 \pm$ 203.78 IU/L before the first treatment session to $58.93 \pm 93.96 \mathrm{IU} / \mathrm{L}$ after the final treatment session when they achieved complete response. PTM: pretibial myxedema; TRAb: TSH receptor antibodies; TSH: thyroid-stimulating hormone.

serum TRAb level at 2 weeks after lesions recurred. Therefore, elevated serum TRAb levels increased the probability of PTM recurrence. Local risk factors further increased the probability of PTM recurrence under the background of TSHR autoimmunity.

\section{Discussion}

This study has obtained the results that further our understand- ing of etiology, diagnosis, treatment and relapse for PTM through observations and treatments of 400 PTM cases over the course of 7 years.

\section{Etiology}

In the case-control study, we investigated 19 factors that were previously suspected. Genetic factors were not included in this study since we did not find any PTM cases with PTM family

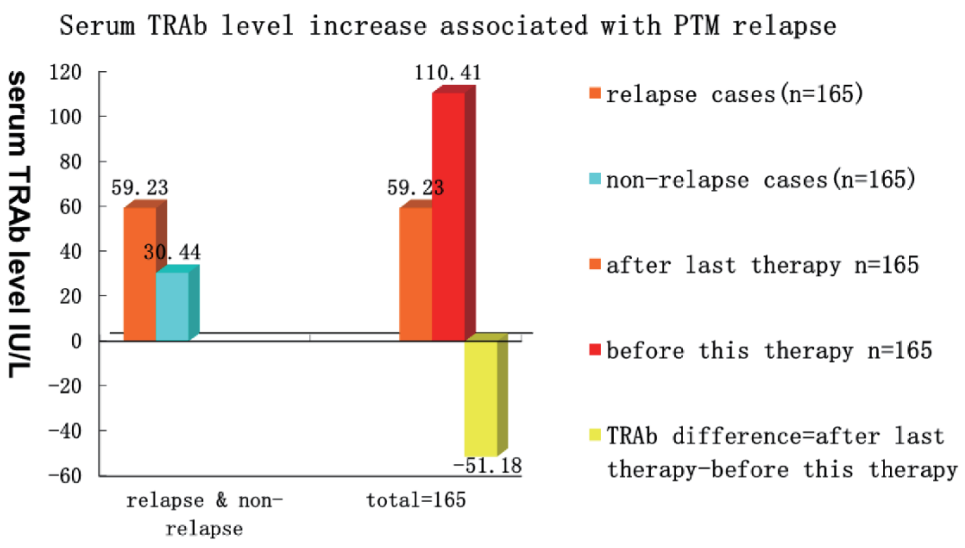

Figure 7. PTM relapses related to increase of serum TRAb levels after stopping glucocorticoid treatment. In 330 cases with complete response, serum TRAb levels $(59.85 \pm 81.12 \mathrm{IU} / \mathrm{L})$ in relapse cases were higher than those $(30.44 \pm 62.10 \mathrm{IU} / \mathrm{L})$ in non-relapse cases $(\mathrm{n}=330, t=2.14, \mathrm{P}=0.033)$. In 165 relapse cases, serum TRAb levels increased by $51.18 \pm 115.99 \mathrm{IU} / \mathrm{L}$ from complete lesion regression (TRAb, $59.23 \pm 70.06 \mathrm{IU} / \mathrm{L})$ after therapy to the first relapse (TRAb, $110.41 \pm 123.78 \mathrm{IU} / \mathrm{L})(\mathrm{n}=$ 165 , paired $t=-3.796, \mathrm{P}<0.001)$. PTM: pretibial myxedema; TRAb: TSH receptor antibodies; TSH: thyroid-stimulating hormone. 
Table 3. Comparison of Local Factors Precipitating PTM Relapse in Different Serum TRAb Levels

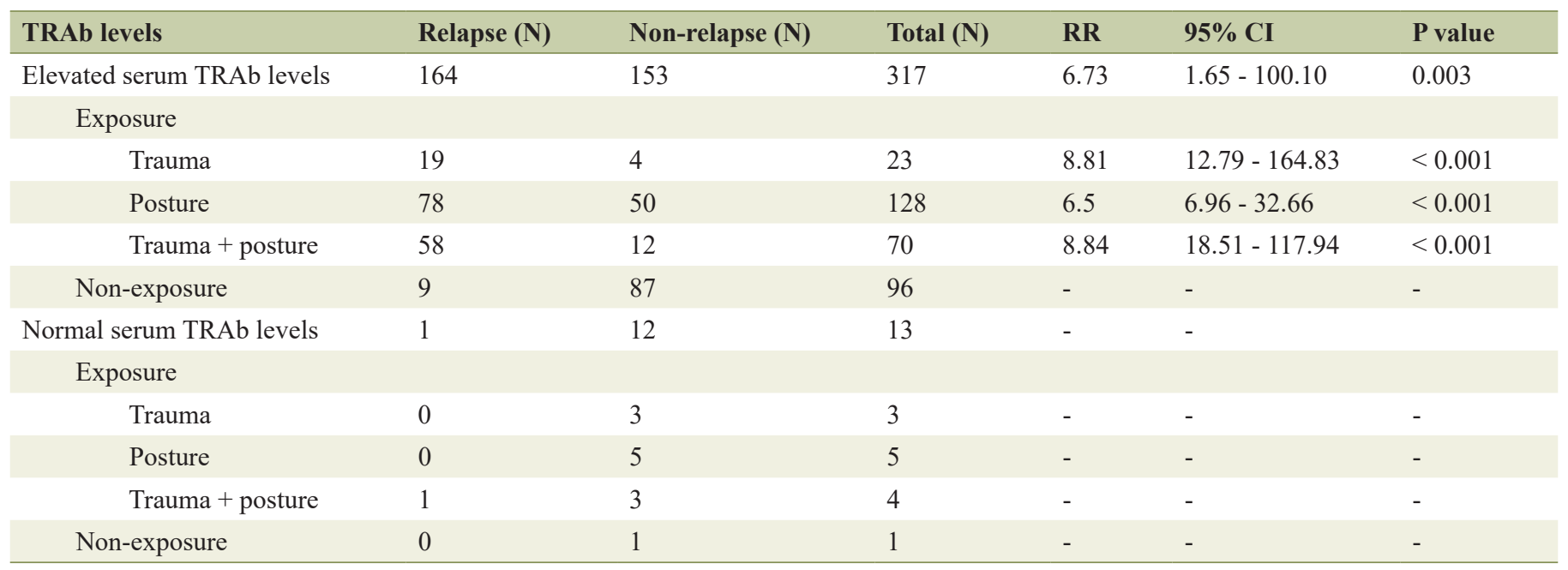

RR: relative risk or risk ratio or rate ratio; $R R>1$ : positive association between exposure factors and PTM relapse; $R R=1.0-1.1$, no association between exposure factors and PTM relapse; $R R=1.2-1.4$, the association is weak; $R R=1.5-2.9$, the association is moderate; $R R=3.0-9.0$, the association is strong; RR $\geq 10$, the association is very strong. PTM: pretibial myxedema; TRAb: TSH receptor antibodies; TSH: thyroid-stimulating hormone.

history. Our results showed that TRAb, AITD or AITD history, local trauma, venous stasis posture, cigarette smoking, and age were risk factors for PTM. The other 13 factors were not risk factors of PTM occurrence.

Although $88 \%$ of PTM cases exhibit exophthalmos, and most exophthalmos occurs before PTM, improving exophthalmos of GO does not influence PTM occurrence [17]. PTM has been reported to occur in $15 \%$ of patients with GD without ophthalmopathy [18]. PTM also can initially present in GD before exophthalmos. In the 400 cases, PTM was the initial presentation in five cases. Finally, exophthalmos can be caused by thyroid diseases and non-thyroid diseases. These phenomena support the conclusion that exophthalmos is not a risk factor for PTM development; whereas thyroid autoimmunity causing GO could be a risk factor for PTM. Thyroid-associated acropachy often occurs after PTM and thus is not its cause. Thyroid dysfunction, exophthalmos, PTM, and acropachy may occur concurrently or successively in AITD, because these disorders share autoantigen TSHR and autoimmunity, and TRAb might precede thyroid dysfunction by many years [19]. Their relationships are overlapping rather than causal. I-131 treatment for hyperthyroidism was suspected as the cause of PTM, because many PTM cases occur after I-131 treatment for GD. However, many PTM cases occur without any treatment. In this study, 245 (61.3\%) cases had I-131 treatment history in the GD w/o PTM group. There was no statistical significance between the two groups by multivariate logistic regression analysis. Taken together, exophthalmos, acropachy, and I-131 treatment were not risk factors but confounding factors for PTM.

Sex ratio $(\mathrm{M} / \mathrm{F})$ of the PTM group in this study was 1:0.91, which is similar to the sex ratio (1:0.96) of the Chinese population in 2018. Sex ratio in our previous report was 1:3.7 (M/F) in PTM patients. This might be because male PTM patients had more serious cases than the females, so more male PTM patients came to see dermatologists. It is also possible that real life data in the hospital are commonly different from epidemiological data [20].

Age was a risk factor for PTM, as older people are more likely to suffer from it. The youngest onset age of PTM has been reported to be 10 years old [21]; it was 15 years old in our previous report and 16 years old in this study. Age might influence the effect of TRAbs on gland function and relapserisk [22]. Perhaps, microcirculation in vessels worsens with age (like varicose veins occurring in the teens and later), or maybe adults are more apt to keep prolonged standing or sitting postures because of occupational requirements.

Cigarette smoking has been thought to be a risk factor for thyroid and extra-thyroid manifestations in GD [9, 23]. Both cigarette smoke extracts and hypoxia have been reported to increase hyaluronic acid production by orbital fibroblasts [24]. Cigarette smoke extracts cause generalized stimulation of the autoimmune process and hypoxia in body tissue.

TRAb, local trauma, venous stasis posture, and AITD or AITD history were found to be risk factors for PTM in the case-control study $[25,26]$. However, risk factors are not equal to the actual causes of PTM. AITD or AITD history itself could not cause PTM, but its cause, i.e., thyroid autoimmunity, could cause extra-thyroid manifestations, such as exophthalmos, PTM, and acropachy. Therefore, AITD or AITD history reflects the existence of thyroid autoimmunity in PTM patients. TPOAb, TgAb, and TRAb are related to thyroid autoimmunity. The results in this study showed that only TRAb was related to PTM occurrence, while the other two autoantibodies were not. TRAb is a product of TSHR autoimmunity in thyroid autoimmunity, and TRAb, and AITD or AITD history as PTM risk factors very likely reflect this.

To test whether TRAb, local trauma, or venous stasis posture were causes of PTM, an immunosuppressive glucocorticoid treatment trial was first performed for the 400 PTM patients, and then the relapse follow-up of 330 patients with 
complete response was conducted with or without exposure to these three main risk factors. The results showed that TRAb, venous stasis, and local skin trauma were causes of PTM. TRAb increase was the basis of PTM relapse, and local risk factors precipitated PTM relapse under the background of TSHR autoimmunity.

Persistent venous stasis posture might lead to a slower return of post-capillary venules and lymphatics by gravity and then high permeability of the venules. Many TRAb molecules enter the skin of the lower legs and stimulate fibroblasts to produce HA through intracellular PI3K-AKT signaling. Both prolonged venous stasis posture and local trauma would also cause local mild hypoxia and might trigger local anti-TSHR autoimmunity through the initiation of local inflammatory responses, resulting in excessive HA deposition and lymphocyte infiltration [13, 2730]. This hypothesis is supported by case reports in which PTM occurs at the sites of cutaneous scars after trauma or surgery and after persistent venous stasis posture [27, 31, 32].

\section{Diagnosis and treatment}

The results in this study revealed that TRAb is not only a cause of PTM but an important feature and biomarker to differentiate it from other mucinoses, such as scleromyxedema, cutaneous focal mucinosis, acral persistent popular mucinosis, stasis mucinosis, and obesity-associated lymphoedematous mucinosis. Thus, $\mathrm{TRAb}$ is an important indicator for the diagnosis of PTM [33].

Multiple modalities of PTM treatment have been reported that include minimizing risk factors (smoking, obesity), topical steroids, intralesional steroids (triamcinolone, betamethasone) [34], plasmapheresis, intravenous immunoglobulin (IVIg), surgery [35], UVA1, compression bandages, and massage therapy. Glucocorticoid inhibiting TSHR autoimmunity and HA synthesis can lower serum TRAb levels and improve PTM $[6,36]$. Rituximab (RTX) and IVIg have recently been reported to achieve improvement or stability of PTM. RTX depletes both B lymphocytes in the intermediate stages of maturation and short-lived plasma cells, whereas IVIg depletes T lymphocytes [37]. This indicates that TSHR autoimmunity of PTM is involved with B and T lymphocytes. We suggest that a PTM treatment program should include avoiding local precipitating factors and blocking TSHR autoimmunity (small blocking TRAb molecules and inhibiting activation of B lymphocytes or CD4 T lymphocytes). This would be suitable not only for PTM but also Graves' orbitopathy and even GD, but further study is needed.

\section{Acknowledgments}

We thank Zaiqing He MD at the Department of Pathology in CNNC 416 Hospital for the technique supports of histopathology. We thank engineers of Computer Network Center, Doctors at Department of Endocrinology and Pediatrics in CNNC 416 Hospital for their supports. We also thank Wei Tang PhD and LetPub (www.letpub.com) for their linguistic assistance of this manuscript.

\section{Financial Disclosure}

This study was supported by Sichuan Science and Technology Department (grant 18YYJCI834), Sichuan Provincial Health Committee (project 110495, project 080141) and Sichuan Education Department (grant 18ZB0173) in Sichuan province.

\section{Conflict of Interest}

All authors do not have any conflict of interest regarding the publication of this manuscript.

\section{Informed Consent}

All subjects provided written informed consent.

\section{Author Contributions}

Changgui Lan: conceptualization, methodology, writing of original draft, review and editing, project administration, and funding acquisition. Liping $\mathrm{Hu}$ : investigation, data curation, software, writing of original draft. Chengqi Liao: investigation, software, writing of original draft, review and editing. Yuhong Shi: investigation, formal analysis, resources, and supervision. Yi Wang: conceptualization, visualization, writing review and editing. Shuanghua Cheng: conceptualization, visualization, and software. Wei Huang: resources, investigation, and software.

\section{Data Availability}

The authors declare that data supporting the findings of this study are available within the article.

\section{References}

1. Watson EM. Localized (Pretibial) Myxoedema. Can Med Assoc J. 1946;54(3):260-265.

2. Taylor S. Graves of Graves' disease, 1796-1853. J R Coll Physicians Lond. 1986;20(4):298-300.

3. Lan C, Chen W, Zhao J, Li C, Mei X, Hu J. Prevalence and clinical characteristics of pretibial myxedema in Chinese outpatients with thyroid diseases. J Endocrinol Metab. 2015;5(4):250-255.

4. Stewart G, Payne CM, Croft DN. Hashimoto's thyroiditis associated with dysthyroid eye disease, pretibial myxoedema and thyroid acropachy. J R Soc Med. 1984;77(3):240-243.

5. Salvi M, De Chiara F, Gardini E, Minelli R, Bianconi L, Alinovi A, Ricci R, et al. Echographic diagnosis of pretibial myxedema in patients with autoimmune thyroid disease. Eur J Endocrinol. 1994;131(2):113-119. 
6. Lan C, Wang Y, Zeng X, Zhao J, Zou X. Morphological diversity of pretibial myxedema and its mechanism of evolving process and outcome: a retrospective study of 216 cases. J Thyroid Res. 2016;2016:2652174.

7. Watson EM, Pearce RH. The cutaneous mucopolysaccharides in localized (pretibial) myxedema. Ann N Y Acad Sci. 1950;52(7):1004-1005.

8. Ando T, Latif R, Davies TF. Thyrotropin receptor antibodies: new insights into their actions and clinical relevance. Best Pract Res Clin Endocrinol Metab. 2005;19(1):33-52.

9. Macchia E, Concetti R, Borgoni F, Cetani F, Fenzi GF, Pinchera A. Assays of TSH-receptor antibodies in 576 patients with various thyroid disorders: their incidence, significance and clinical usefulness. Autoimmunity. 1989;3(2):103-112.

10. Fatourechi V. Pretibial myxedema: pathophysiology and treatment options. Am J Clin Dermatol. 2005;6(5):295309.

11. Cheung HS, Nicoloff JT, Kamiel MB, Spolter L, Nimni ME. Stimulation of fibroblast biosynthetic activity by serum of patients with pretibial myxedema. J Invest Dermatol. 1978;71(1):12-17.

12. Kumar S, Iyer S, Bauer H, Coenen M, Bahn RS. A stimulatory thyrotropin receptor antibody enhances hyaluronic acid synthesis in graves' orbital fibroblasts: inhibition by an IGF-I receptor blocking antibody. J Clin Endocrinol Metab. 2012;97(5):1681-1687.

13. Rapoport B, Alsabeh R, Aftergood D, McLachlan SM. Elephantiasic pretibial myxedema: insight into and a hypothesis regarding the pathogenesis of the extrathyroidal manifestations of Graves' disease. Thyroid. 2000;10(8):685-692.

14. Schwartz KM, Fatourechi V, Ahmed DD, Pond GR. Dermopathy of Graves' disease (pretibial myxedema): longterm outcome. J Clin Endocrinol Metab. 2002;87(2):438446.

15. Menconi F, Marcocci C, Marino M. Diagnosis and classification of Graves' disease. Autoimmun Rev. 2014;13(45):398-402.

16. Ross DS, Burch HB, Cooper DS, Greenlee MC, Laurberg P, Maia AL, Rivkees SA, et al. 2016 American Thyroid Association Guidelines for diagnosis and management of hyperthyroidism and other causes of thyrotoxicosis. Thyroid. 2016;26(10):1343-1421.

17. Fatourechi V, Pajouhi M, Fransway AF. Dermopathy of Graves disease (pretibial myxedema). Review of 150 cases. Medicine (Baltimore). 1994;73(1):1-7.

18. Lohiya S, Lohiya V, Stahl EJ. Pretibial myxedema without ophthalmopathy: an initial presentation of Graves' disease. Am J Med Sci. 2013;346(1):73-75.

19. Muller I, Willis M, Healy S, Nasser T, Loveless S, Butterworth S, Zhang L, et al. Longitudinal characterization of autoantibodies to the thyrotropin receptor (TRAb) during alemtuzumab therapy: evidence that TRAb may precede thyroid dysfunction by many years. Thyroid. 2018;28(12):1682-1693.

20. Hussain YS, Hookham JC, Allahabadia A, Balasubramanian SP. Epidemiology, management and outcomes of Graves' disease-real life data. Endocrine. 2017;56(3):568-
578.

21. Kraus CN, Sodha P, Vaidyanathan P, Kirkorian AY. Thyroid dermopathy and acropachy in pediatric patients. Pediatr Dermatol. 2018;35(6):e371-e374.

22. Bano A, Gan E, Addison C, Narayanan K, Weaver JU, Tsatlidis V, Razvi S. Age May influence the impact of TRAbs on thyroid function and relapse-risk in patients with Graves disease. J Clin Endocrinol Metab. 2019;104(5):1378-1385.

23. Bahn RS. Current insights into the pathogenesis of Graves' ophthalmopathy. Horm Metab Res. 2015;47(10):773778.

24. Kahaly GJ, Olivo PD. Graves' Disease. N Engl J Med. 2017;376(2):184.

25. Kriss JP, Pleshakov V, Chien JR. Isolation and identification of the long-acting thyroid stimulator and its relation to hyperthyroidism and circumscribed pretibial myxedema. J Clin Endocrinol Metab. 1964;24:1005-1028.

26. Pimstone BL, Hoffenberg R, Black E. Parallel Assays of Thyrotrophin, Long-acting thyroid stimulator and exophthalmos-producing substance in endocrine exophthalmos and pretibial myxedema. J Clin Endocrinol Metab. 1964;24:976-982.

27. Liao $\mathrm{C}, \mathrm{Hu} \mathrm{L}$, Lan $\mathrm{C}$. Hashimoto's thyroiditis associated with an extra-thyroidal triad of pretibial myxedema, ophthalmopathy, and acropachy. J Biol Regul Homeost Agents. 2020;34(3):1085-1090.

28. Zheng J, Song F, Lu SL, Wang XQ. Dynamic hypoxia in scar tissue during human hypertrophic scar progression. Dermatol Surg. 2014;40(5):511-518.

29. Raffetto JD. Pathophysiology of chronic venous disease and venous ulcers. Surg Clin North Am. 2018;98(2):337347.

30. Kondo T, Ishida Y. Molecular pathology of wound healing. Forensic Sci Int. 2010;203(1-3):93-98.

31. Tong DW, Ho KK. Pretibial myxoedema presenting as a scar infiltrate. Australas J Dermatol. 1998;39(4):255-257.

32. Missner SC, Ramsay EW, Houck HE, Kauffman CL. Graves' disease presenting as localized myxedema in a thigh donor graft site. J Am Acad Dermatol. 1998;39(5 Pt 2):846-849.

33. Kahaly GJ, Diana T, Olivo PD. Tsh receptor antibodies: relevance \& utility. Endocr Pract. 2020;26(1):97-106.

34. Lan C, Li C, Chen W, Mei X, Zhao J, Hu J. A Randomized Controlled Trial of Intralesional Glucocorticoid for Treating Pretibial Myxedema. J Clin Med Res. 2015;7(11):862-872.

35. Lan C, Li C, Yang M, Mei X, He Z, Chen W, Chen H, et al. Pretibial myxoedema with autoimmunity and hyperplasia treated with glucocorticoids and surgery. Br J Dermatol. 2012;166(2):457-459.

36. Gebhardt C, Averbeck M, Diedenhofen N, Willenberg A, Anderegg U, Sleeman JP, Simon JC. Dermal hyaluronan is rapidly reduced by topical treatment with glucocorticoids. J Invest Dermatol. 2010;130(1):141-149.

37. Kotwal A, Turcu AF, Sonawane V, Bahn RS, Pittelkow MR, Bridges A, Stan MN. Clinical experience with rituximab and intravenous immunoglobulin for pretibial myxedema: a case series. Thyroid. 2019;29(5):692-699. 